\title{
Monitoramento do nitrogênio na planta e no solo para predição da adubação nitrogenada em milho
}

\author{
Lisandro Rambo ${ }^{(1)}$, Paulo Regis Ferreira da Silva(2), Mércio Luiz Strieder ${ }^{(2)}$, Luís Sangoi ${ }^{(3)}$, Cimélio Bayer ${ }^{(4)}$ \\ e Gilber Argenta ${ }^{(1)}$
}

\begin{abstract}
(1)Syngenta Seeds Ltda., Rod. BR-452, Km 142, Caixa Postal 585, CEP 38400-974 Uberlândia, MG. E-mail: lisandro.rambo@syngenta.com, gilber.argenta@syngenta.com (2)Universidade Federal do Rio Grande do Sul (UFRGS), Fac. de Agronomia (FA), Dep. de Plantas de Lavoura, Av. Bento Gonçalves, 7712, Caixa Postal 15.100, CEP 90970-100 Porto Alegre, RS. E-mail: paulo.silva@ufrgs.br, domercio@gmail.com (3) Universidade Estadual de Santa Catarina, Dep. de Fitotecnia, Caixa Postal 281, CEP 88520-000 Lages, SC. E-mail: a2ls@cav.udesc.br (4)UFRGS, FA, Dep. de Solos. E-mail: cimelio.bayer@ufrgs.br
\end{abstract}

Resumo - O objetivo deste trabalho foi comparar o monitoramento do teor de $\mathrm{N}$ na planta com base no índice do teor relativo crítico de clorofila na folha (TRCC) e no índice de suficiência (IS), determinados pelo clorofilômetro, e avaliar a integração de características de planta e de solo no monitoramento de N no sistema solo-planta em milho. Foram conduzidos dois experimentos em Eldorado do Sul, RS. Os tratamentos foram sistemas de manejo de $\mathrm{N}$, que diferiram quanto à época, dose e critério para decidir pela aplicação de $\mathrm{N}$ (com monitoramento e sem monitoramento). Nos sistemas monitorados, o TRCC e o IS foram usados para indicar a época de aplicação de $\mathrm{N}$ em cobertura e testou-se o monitoramento integrado, usando o TRCC e o teor de nitrato no solo. O rendimento e os incrementos no rendimento de grãos e na margem bruta, bem como as eficiências técnica, econômica e de uso do $\mathrm{N}$ foram maiores nos sistemas monitorados que nos sistemas sem monitoramento. A eficiência do monitoramento, com base no TRCC e no IS e na intregração entre o teor de nitrato no solo e o TRCC, foi similar. O monitoramento com base na integração entre o teor de nitrato no solo e o TRCC não incrementou a eficiência de uso do N no ambiente avaliado.

Termos para indexação: Zea mays, época de aplicação, rendimento de grãos, eficiência de uso de nitrogênio, clorofilômetro.

\section{Monitoring plant and soil nitrogen status to predict nitrogen fertilization in corn}

\begin{abstract}
The objective of this work was to compare the monitoring of corn plant nitrogen status using two indexes of leaf relative chlorophyll content (critical leaf relative chlorophyll content - CRCC and sufficiency index - SI) determinated by chlorophyll meter and to evaluate the integration between plant and soil characteristics for monitoring the $\mathrm{N}$ content of the soil-plant system in corn. Two field experiments were conducted in Eldorado do Sul, State of Rio Grande do Sul, Brazil. The treatments were different N management systems, with different $\mathrm{N}$ application times and $\mathrm{N}$ rates, carried out with or without monitoring. In the monitored systems, CRCC and SI were used to indicate when $\mathrm{N}$ side-dress should be performed and it was tested, using the CRCC and soil nitrate content, one integrated monitoring system. Grain yield and grain yield increase, gross profit increase and the technical, economic and $\mathrm{N}$ use efficiency were often higher in the monitored systems than in the systems without monitoring. The efficiency of the monitoring methods, using the CRCC and using the SI and integrating the CRCC and soil nitrate content, was similar. Monitoring corn N status integrating the CRCC and soil nitrate content did not improve nitrogen use efficiency in the tested environment.
\end{abstract}

Index terms: Zea mays, application time, grain yield, nitrogen use efficiency, chlorophyll meter.

\section{Introdução}

A eficiência mundial estimada de uso do N (EUN) em cereais é de apenas 33\%. Considerando os $67 \%$ de $\mathrm{N}$ que não são aproveitados, tem-se uma perda anual de 15,9 bilhões de dólares em fertilização nitrogenada (Raun \& Johnson, 1999), além dos prováveis impactos negativos ao ambiente. Uma das alternativas para aumentar a EUN é sincronizar a época de aplicação de $\mathrm{N}$ com a necessidade da planta.
Indicadores de solo e de planta têm sido usados para monitorar a disponibilidade de $\mathrm{N}$ e auxiliar na decisão sobre a dose e a época de sua aplicação. Entre as características de solo utilizadas como indicadores do nível de $\mathrm{N}$ no solo, tem-se destacado o teor de $\mathrm{N}$ mineral, especialmente o de nitrato. $\mathrm{O}$ uso do nitrato é facilitado pela disponibilidade de testes rápidos para sua determinação (Roth et al., 1991; Sims et al., 1995) e pelo fato de que parte do $\mathrm{N}$ mineral no solo está sob a forma de nitrato (Sims et al., 1995; Ma \& Dwyer, 1999). 
Entre as características da planta, destaca-se a determinação do teor relativo de clorofila na folha (TRC) pelo clorofilômetro, que tem sido bastante estudado nos últimos anos, em virtude de sua rapidez, precisão e baixo custo. O clorofilômetro, como o Minolta SPAD-502, permite obtenção de valores indiretos do teor de clorofila presente na folha, de modo não destrutivo, rápido e simples. Este método fundamenta-se na correlação positiva existente entre teor de clorofila na folha e teor de $\mathrm{N}$ na planta (Waskom et al., 1996; Argenta et al., 2004) que, por sua vez, está associada à atividade fotossintética (Ma et al., 1995). Devido a estas vantagens, e por estar correlacionado com rendimento de grãos, a determinação do TRC pelo clorofilômetro tem sido usada para predizer a necessidade de adubação nitrogenada na cultura do milho (Varvel et al., 1997; Costa et al., 2001; Argenta et al., 2004).

$\mathrm{O}$ uso do clorofilômetro no manejo da adubação nitrogenada em milho vem sendo estudado sob duas abordagens: a de valores críticos do teor relativo de clorofila (TRCC) e do índice de suficiência de N (IS). A utilização de valores críticos pressupõe que, para cada estádio de desenvolvimento, haja um valor mínimo do TRC (nível crítico - TRCC) que a planta apresenta de acordo com leitura do clorofilômetro, correspondente ao nível mínimo de clorofila necessário, naquela fase da ontogenia, para maximizar o rendimento de grãos. Leituras abaixo deste nível indicam necessidade de suplementação de $\mathrm{N}$ pela adubação, ao passo que as acima do valor crítico indicam que não é recomendada a fertilização.

Estudos têm determinado valores críticos durante a ontogenia da cultura do milho (Jemison \& Lytle, 1996; Sunderman et al., 1997; Argenta et al., 2004). Argenta et al. (2004) elaboraram uma curva do teor adequado de $\mathrm{N}$ para o milho cultivado sob condições de fertilização elevada, controle de plantas daninhas, pragas e doenças adequado e com irrigação, a partir de níveis críticos determinados em quatro estádios de desenvolvimento, em função dos valores de TRC lidos pelo clorofilômetro. Esses valores aumentaram de forma quadrática à medida que a planta avançou no seu desenvolvimento, o que corrobora resultados obtidos por Sunderman et al. (1997). Pela equação estimada, pode-se fazer a extrapolação dos valores de leitura do aparelho para os estádios intermediários aos que foram avaliados.
Outros autores têm recomendado um método de calibração das leituras do clorofilômetro para cada área de cultivo, híbrido, estádio de desenvolvimento, condição edafoclimática e prática de manejo (Waskom et al., 1996; Hussain et al., 2000; Costa et al., 2001). Uma forma de calibrar as leituras é a determinação do índice de suficiência (IS), que é obtido pela média das leituras do clorofilômetro nas amostras, dividida pela média das leituras do aparelho na área de referência. Esta área de referência é uma faixa da lavoura, com a mesma cultivar, adubada com uma dose de $\mathrm{N}$ bem superior à do restante da lavoura, de forma que, teoricamente, não ocorra deficiência. $\mathrm{O}$ uso dessa dose elevada de $\mathrm{N}$ na faixa de referência é possível porque o TRC não está associado ao consumo de luxo de N, sob forma de nitrato (Blackmer \& Schepers, 1994, 1995). O valor que tem sido mais utilizado como IS é igual a 0,95. Assim, quando o percentual relativo de clorofila da amostra situar-se abaixo de $95 \%$ da leitura, na faixa de referência, recomenda-se a aplicação da adubação nitrogenada (Blackmer \& Schepers, 1994; Waskom et al., 1996; Varvel et al., 1997).

Apesar de existirem estudos sobre o uso dos índices do TRCC e do IS no manejo da adubação nitrogenada em cobertura em milho, não há trabalhos que tenham comparado a eficiência de uso desses dois índices no monitoramento do teor de $\mathrm{N}$ na planta para predizer a sua necessidade de aplicação. Da mesma forma, não se conhecem estudos que tenham testado o uso de características de planta e de solo de forma integrada para monitoramento do nível de N no sistema solo-planta.

O objetivo deste trabalho foi comparar a eficiência do monitoramento do nível de $\mathrm{N}$ na planta com base em dois índices de teor relativo de clorofila na folha (TRCC e IS), determinados pelo clorofilômetro, como indicadores do manejo da adubação nitrogenada em cobertura em milho, e testar a possibilidade de uso integrado de características de planta e de solo no monitoramento do nível de $\mathrm{N}$ do sistema solo-planta para auxiliar no manejo da adubação nitrogenada.

\section{Material e Métodos}

Foram conduzidos dois experimentos em campo, em áreas adjacentes, no Município de Eldorado do Sul, região ecoclimática da Depressão Central do Estado do Rio Grande do Sul, nas estações de crescimento 2002/ 2003 (Experimento I) e 2003/2004 (Experimento II). 
O solo da área experimental é classificado como Argissolo Vermelho distrófico típico (Embrapa, 1999). Os resultados da análise de solo no ano de 2002/2003 foram: $290 \mathrm{~g} \mathrm{~kg}^{-1}$ de argila; $\mathrm{pH}$ (água) 5,4; 5,9 de índice SMP; 9,4 mg dm${ }^{-3}$ de P; $105 \mathrm{mg} \mathrm{dm}{ }^{-3}$ de K; $25 \mathrm{~g} \mathrm{~kg}^{-1}$ de MO e 7,6 $\mathrm{cmol}_{\mathrm{c}} \mathrm{dm}^{-3} \mathrm{de}$ CTC. No ano de 2003/2004, os resultados foram: $290 \mathrm{~g} \mathrm{~kg}^{-1}$ de argila; $\mathrm{pH}$ (água) 5,3; 6,2 de índice SMP; $5,6 \mathrm{mg} \mathrm{dm}^{-3} \mathrm{de} \mathrm{P} ; 161 \mathrm{mg} \mathrm{dm}^{-3} \mathrm{de}$ $\mathrm{K} ; 25 \mathrm{~g} \mathrm{~kg}^{-1}$ de MO e $8,1 \mathrm{cmol}_{\mathrm{c}} \mathrm{dm}^{-3}$ de CTC.

Os tratamentos nos experimentos I e II constaram de 10 e 14 sistemas de manejo de $\mathrm{N}$, respectivamente, agrupados de acordo com a dose máxima de $\mathrm{N}$ aplicada para facilitar a análise e compreensão dos resultados. A definição dos grupos de sistemas de manejo de $\mathrm{N}$ em dose média, alta e muita alta foi estipulada pela quantidade máxima de $\mathrm{N}$ aplicada de 130, 260 e $455 \mathrm{~kg} \mathrm{ha}^{-1}$, respectivamente, com base nas recomendações da pesquisa oficial no Estado do Rio Grande do Sul para a cultura do milho (Indicações..., 2005) e trabalho desenvolvido por Amado et al. (2002).

\section{Experimento I}

O híbrido simples, de ciclo superprecoce, Pioneer 32R21 foi semeado no dia 14 de outubro de 2002, em semeadura direta, em sucessão ao consórcio aveiapreta e ervilhaca, na densidade de 65.000 plantas $^{-1} \mathrm{a}^{-1}$ e espaçamento entrelinhas de $0,7 \mathrm{~m}$. A adubação com $\mathrm{P}$ e $\mathrm{K}$ foi realizada na linha, por ocasião da semeadura, constando de $80 \mathrm{~kg} \mathrm{ha}^{-1}$ de $\mathrm{P}_{2} \mathrm{O}_{5}$ e $60 \mathrm{~kg} \mathrm{ha}^{-1}$ de $\mathrm{K}_{2} \mathrm{O}$, de acordo com a análise de solo. O experimento foi irrigado por aspersão, quando o potencial de água no solo era inferior a $-0,04 \mathrm{MPa}$.

O delineamento experimental foi o de blocos ao acaso, com quatro repetições. Os tratamentos constaram de dez sistemas (S) de manejo de N que foram S2, S3 e S4 no grupo dose média, S5, S6 e S7 no grupo dose alta e S8, S9 e S10 no grupo dose muito alta. Em cada grupo, foi incluído o sistema $\mathrm{S} 1$ (sem N) como testemunha.

Os sistemas $\mathrm{S} 1, \mathrm{~S} 4, \mathrm{~S} 7$ e $\mathrm{S} 10$ corresponderam às doses de $\mathrm{N}$ de $0,130,260$ e $455 \mathrm{~kg} \mathrm{ha}^{-1}$, respectivamente, aplicadas na semeadura e em quatro estádios de desenvolvimento, exceto no sistema S1. Na semeadura, foram aplicadas as doses de 10, $20 \mathrm{e} 35 \mathrm{~kg} \mathrm{ha}^{-1}$, correspondendo, respectivamente, aos sistemas $\mathrm{S} 4, \mathrm{~S} 7$ e S10, denominados sistemas padrões. No estádio V3, foram aplicadas as doses em cobertura de 30,60 e $105 \mathrm{~kg} \mathrm{ha}^{-1}$ nos sistemas $\mathrm{S} 4, \mathrm{~S} 7$ e S10, respectivamente. O restante do $\mathrm{N}$ em cobertura foi aplicado em três doses iguais, nos estádios
V6, V10 e espigamento. O N em cobertura foi aplicado ao lado das plantas na linha, usando como fonte a uréia.

Os sistemas de manejo S2, S5 e S8 equivaleram aos sistemas S4, S7 e S10, com a diferença de que foram monitorados com clorofilômetro e as doses de $\mathrm{N}$ foram aplicadas somente quando os valores lidos eram inferiores aos níveis críticos estabelecidos por Argenta (2001), nos quais as leituras no clorofilômetro de 45,4,52,1, 55,3, e 58 correspondem ao nível crítico, nos estádios V3, V6, V10 e espigamento, respectivamente. Os sistemas S3, S6 e S9 também foram monitorados com clorofilômetro, assim como os sistemas S2, S5 e S8. No entanto, esse monitoramento teve como base o IS de 0,95 . O IS foi determinado a partir dos valores do TRC na folha obtidos nos sistemas S3, S6 e S9, divididos pelo valor do TRC na folha obtido na parcela com o nível mais alto de $\mathrm{N}$ (parcela de referência). As doses de $\mathrm{N}$ preestabelecidas para os sistemas S3, S6 e S9 foram iguais às dos sistemas S4, S7 e S10, para cada estádio de desenvolvimento, sendo aplicadas somente quando o valor do IS era inferior a 0,95.

\section{Experimento II}

A semeadura direta do híbrido pioneer 32R31 foi realizada no dia 25 de setembro de 2003, em sucessão à aveia-preta, na mesma densidade e espaçamento do Experimento I. A adubação com $\mathrm{P}$ e $\mathrm{K}$ foi realizada da mesma forma, porém utilizando-se as doses de $105 \mathrm{~kg} \mathrm{ha}^{-1}$ de $\mathrm{P}_{2} \mathrm{O}_{5}$ e $105 \mathrm{~kg} \mathrm{ha}^{-1}$ de $\mathrm{K}_{2} \mathrm{O}$, de acordo com a análise de solo. A irrigação seguiu os mesmos critérios do Experimento I.

Os tratamentos constaram de 14 sistemas (S) de manejo de N, que foram S2, S3, S4 e S11 no grupo dose média, S5, S6, S7 e S12 no grupo dose alta e S8, S9, S10 e S13 no grupo dose muito alta. Em cada grupo, foi incluído o sistema S1 como testemunha e o sistema S14 como referência (sem limitação de $\mathrm{N}$ ). $\mathrm{O}$ delineamento experimental foi o de blocos ao acaso, com quatro repetições. Neste experimento, os sistemas S1 (sem N), S14 (parcela de referência), S4, S7 e S10 representam níveis de $\mathrm{N}$ de 0 , $555,130,260$ e $455 \mathrm{~kg} \mathrm{ha}^{-1}$, respectivamente. As doses de $\mathrm{N}$ foram aplicadas na semeadura e em quatro estádios de desenvolvimento, exceto no sistema S1. Na semeadura, foram aplicadas as doses de 10,20 e $35 \mathrm{~kg} \mathrm{ha}^{-1}$, correspondendo, respectivamente, aos sistemas S4, S7 e S10, denominados padrões. No estádio V3, foram aplicadas as doses de 30, 60 e $105 \mathrm{~kg} \mathrm{ha}^{-1}$ nos sistemas S4, S7 e S10, respectivamente. $\mathrm{O}$ restante do $\mathrm{N}$ foi aplicado em três doses iguais, nos estádios V6, V10 e espigamento. 
Os sistemas de manejo S2, S5 e S8 equivaleram aos sistemas S4, S7 e S10, com a diferença de que foram monitorados com clorofilômetro e as doses de $\mathrm{N}$ foram aplicadas somente quando os valores lidos foram inferiores aos níveis críticos (TRCC) estabelecidos por Argenta (2001), correspondentes às leituras no clorofilômetro de $45,4,52,1,55,3$ e 58,0, para os estádios V3, V6, V10 e espigamento, respectivamente. Os sistemas S3, S6 e S9 também foram monitorados com clorofilômetro, assim como os sistemas S2, S5 e S8. No entanto, este monitoramento teve como base o IS de 0,95 , que foi determinado a partir dos valores do teor relativo de clorofila (TRC) na folha obtidos nos sistemas S3, S6 e S9, divididos pelo valor do TRC na folha obtido na parcela com a maior quantidade de $\mathrm{N}$ aplicado (parcela de referência S14). As doses de $\mathrm{N}$ preestabelecidas para os sistemas $\mathrm{S} 3$, S6 e S9 foram iguais às dos sistemas S4, S7 e S10, em cada estádio de desenvolvimento. Contudo, essas doses foram aplicadas somente quando o valor do IS era inferior ao valor de 0,95 . Os sistemas $\mathrm{S} 11, \mathrm{~S} 12$ e S13 foram monitorados segundo o TRCC, da mesma forma que os sistemas S2, S5 e S8, exceto no estádio V6, cujo monitoramento foi realizado com base na determinação do teor de nitrato no solo, utilizando como valor crítico $20,3 \mathrm{mg} \mathrm{kg}^{-1}$. Este valor foi determinado em um experimento preliminar.

As determinações realizadas no milho foram: teor relativo de clorofila na folha (TRC), teor de $\mathrm{N}$ na planta e rendimento de grãos. O TRC, medido com o clorofilômetro, modelo Minolta SPAD-502, em cinco plantas por parcela, foi determinado nos estádios V3, V6, V10 e espigamento. As leituras com o medidor de clorofila (duas por folha) foram realizadas em pontos situados na metade a dois terços do comprimento da folha, a partir da base, e a dois centímetros da sua margem. Os valores obtidos pelo clorofilômetro foram usados para cálculo do IS.

No Experimento I, considerou-se como parcela de referência aquela com valores mais altos do TRC em cada estádio de desenvolvimento. Desta forma, a parcela de referência não foi a mesma nos estádios de desenvolvimento avaliados. Quanto ao Experimento II, foi prevista a implantação de uma parcela de referência especificamente para determinação do IS. O teor de $\mathrm{N}$ na planta foi determinado a partir de uma amostra de cinco plantas inteiras por parcela, coletadas na colheita, seguindo os procedimentos de Tedesco et al. (1995). O rendimento de grãos foi obtido pela extrapolação da produção da área útil da parcela $\left(8,4 \mathrm{~m}^{2}\right)$ para um hectare, corrigindo-se a umidade para $130 \mathrm{~g} \mathrm{~kg}^{-1}$. Além das avaliações anteriores, no Experimento II foi determinado, no estádio V6, o teor de nitrato no solo de 0 a $30 \mathrm{~cm}$ de profundidade nos sistemas S11, S12 e S13, conforme os procedimentos de Tedesco et al. (1995).

Nos dois experimentos, também foram calculados os aumentos no rendimento de grãos, na margem bruta e as eficiências técnica, econômica e de uso de N. O aumento no rendimento de grãos foi calculado pela diferença entre o rendimento de grãos das parcelas que receberam e das que não receberam adubação nitrogenada. $\mathrm{O}$ aumento na margem bruta foi calculado pela diferença entre o lucro obtido com o aumento no rendimento de grãos decorrente da aplicação de $\mathrm{N}$ ( $\mathrm{R} \$ 17,00$ por $60 \mathrm{~kg}$ de milho) e o custo do fertilizante ( $R$ \$ 785,00 por t de uréia) e de sua aplicação ( $R$ \$ 18,13 por aplicação), de acordo com Federação das Cooperativas Agropecuárias do Rio Grande do Sul (2007). A eficiência técnica de $\mathrm{N}$ foi calculada pela expressão: [(rendimento de grãos do tratamento com $\mathrm{N}$ - rendimento de grãos da testemunha sem $\mathrm{N}$ )/dose de $\mathrm{N}$ aplicada]. A eficiência econômica de $\mathrm{N}$ foi determinada pela expressão: receita bruta advinda do incremento da produtividade com a aplicação de N/custos do fertilizante e de sua aplicação. A eficiência de uso de N (EUN) foi calculada pela expressão: (rendimento de grãos da parcela fertilizada com $\mathrm{N}$ - rendimento de grãos da testemunha sem $\mathrm{N}) /(\mathrm{N}$ acumulado na planta do tratamento com $\mathrm{N}$ - $\mathrm{N}$ acumulado na planta da testemunha sem $\mathrm{N}$ ).

A análise estatística foi realizada em cada grupo de tratamentos por dose de $\mathrm{N}$ (dose média, alta e muito alta) para cada experimento. Optou-se pela análise por grupo porque, em cada um deles havia um sistema padrão (não monitorado), que serviu como referência para comparação com os sistemas monitorados. A formação dos grupos foi efetuada em função das doses máximas de $\mathrm{N}$ aplicadas. Os dados obtidos foram submetidos à análise de variância pelo teste $\mathrm{F}$. A comparação entre médias foi realizada pelo teste de Duncan, a 5\% de probabilidade.

\section{Resultados e Discussão}

No Experimento I (Tabela 1), o teor relativo de clorofila na folha (TRC), em V3 e V6, nos sistemas monitorados S2, S5 e S8 foram inferiores, respectivamente, aos teores críticos de 45,4 e 52,1 estabelecidos por Argenta (2001). Os valores do 
índice de suficiência (IS) também foram menores que o do valor crítico de 0,95 , em todos os sistemas monitorados com este índice, exceto no sistema S9 no estádio V6. No estádio V10, os valores de TRC nos sistemas monitorados S2, S5 e S8 foram inferiores ao do TRCC de 55,3, determinado por Argenta (2001). Já nos sistemas monitorados de acordo com o IS (S3, S6 e S9), os valores obtidos foram superiores a 0,95 . No espigamento, os sistemas S5 e S8 apresentaram valores do TRC superiores ao do TRCC de 58, determinado por Argenta (2001). Nos sistemas S6 e S9, monitorados de acordo com o IS, os valores obtidos foram maiores que 0,95 .

No Experimento II (Tabela 2), novamente os valores de TRC nos estádios V3 e V6 nos sistemas monitorados conforme o TRCC (S2, S5, S8, S11, S12 e S13) foram inferiores, respectivamente, aos dos valores críticos de
45,4 e 52,1, estabelecidos por Argenta (2001). Nestes estádios, os valores de IS também foram menores que 0,95, em dois dos sistemas monitorados com este índice (S3 e S6), exceto no sistema S9, no estádio V6, em que o IS obtido foi superior a 0,95 . Os teores de nitrato no solo no estádio V6 nos sistemas S11, S12 e S13 foram inferiores ao valor crítico de $20,3 \mathrm{mg} \mathrm{kg}^{-1}$. No estádio V10, os valores de TRC em todos os sistemas monitorados de acordo com o TRCC foram inferiores ao valor crítico de 55,3. Os valores de IS também foram menores que 0,95 , em dois dos sistemas monitorados com este índice (S3, S9), mas foi maior no sistema S6. No estádio de espigamento, em quatro dos sistemas monitorados (S5, S8, S12 e S13), os valores do TRC foram superiores ao do nível crítico de 58, ao passo que em dois (S2 e S11) foram inferiores. Quanto aos sistemas monitorados com base no IS, todos os valores nesse estádio foram inferiores a 0,95 .

Tabela 1. Teor relativo de clorofila na folha (TRC) do híbrido de milho Pioneer 32R21, índice de suficiência de N (IS) e doses de $\mathrm{N}$ aplicadas em dez sistemas de manejo de $\mathrm{N}$, em quatro estádios de desenvolvimento da planta, no experimento I.

\begin{tabular}{|c|c|c|c|c|c|c|c|c|c|c|c|c|c|c|}
\hline \multirow{3}{*}{$\begin{array}{l}\text { Sistemas } \\
\text { de manejo }\end{array}$} & \multicolumn{2}{|c|}{$\mathrm{N}$ total aplicado $\left(\mathrm{kg} \mathrm{ha}^{-1}\right)$} & \multicolumn{12}{|c|}{ Estádios de desenvolvimento } \\
\hline & \multirow[t]{2}{*}{ Semeadura } & \multirow[t]{2}{*}{ Cobertura } & \multicolumn{3}{|c|}{ V3 } & \multicolumn{3}{|c|}{ V6 } & \multicolumn{3}{|c|}{ V10 } & \multicolumn{3}{|c|}{ Espigamento } \\
\hline & & & TRC & IS & $\begin{array}{c}\mathrm{N} \text { aplicado } \\
\left(\mathrm{kg} \mathrm{ha}^{-1}\right)\end{array}$ & TRC & IS & $\begin{array}{c}\mathrm{N} \text { aplicado } \\
\left(\mathrm{kg} \mathrm{ha}^{-1}\right)\end{array}$ & TRC & IS & $\begin{array}{c}\mathrm{N} \text { aplicado } \\
\left(\mathrm{kg} \mathrm{ha}^{-1}\right)\end{array}$ & $\overline{\mathrm{TRC}}$ & IS & $\begin{array}{c}\mathrm{N} \text { aplicado } \\
\left(\mathrm{kg} \mathrm{ha}^{-1}\right)\end{array}$ \\
\hline S1 & 0 & 0 & 32,0 & 0,68 & 0 & 44,8 & 0,83 & 0 & 43,6 & 0,81 & 0 & 43,1 & 0,72 & 0 \\
\hline S2 & 10 & 120 & 37,2 & - & 30 & 50,3 & - & 30 & 52,7 & - & 30 & 57,7 & - & 30 \\
\hline S3 & 10 & 90 & - & 0,77 & 30 & - & 0,94 & 30 & - & 0,96 & 0 & - & 0,90 & 30 \\
\hline S4 & 10 & 120 & - & - & 30 & - & - & 30 & - & - & 30 & - & - & 30 \\
\hline S5 & 20 & 180 & 33,4 & - & 60 & 51,1 & - & 60 & 52,5 & - & 60 & 58,2 & - & 0 \\
\hline S6 & 20 & 120 & - & 0,85 & 60 & - & 0,91 & 60 & - & 0,97 & 0 & - & 0,97 & 0 \\
\hline S7 & 20 & 240 & - & - & 60 & - & - & 60 & - & - & 60 & - & - & 60 \\
\hline S8 & 35 & 315 & 40,7 & - & 105 & 51,5 & - & 105 & 52,7 & - & 105 & 58,4 & - & 0 \\
\hline S9 & 35 & 105 & - & 0,89 & 105 & - & 0,95 & 0 & - & 0,98 & 0 & - & 0,96 & 0 \\
\hline S10 & 35 & 420 & - & - & 105 & - & - & 105 & - & - & 105 & - & - & 105 \\
\hline
\end{tabular}

(1)S1, testemunha; S2, monitorado-TRCC; S3, monitorado-IS; S4, padrão; S5, monitorado-TRCC; S6, monitorado-IS; S7, padrão; S8, monitorado-TRCC; S9, monitorado-IS; S10, padrão.

Tabela 2. Teor relativo de clorofila na folha (TRC) do híbrido de milho Pioneer 32R21, índice de suficiência de N (IS) e doses de $\mathrm{N}$ aplicadas em 14 sistemas de manejo de $\mathrm{N}$, em quatro estádios de desenvolvimento da planta, no experimento II.

\begin{tabular}{|c|c|c|c|c|c|c|c|c|c|c|c|c|c|c|}
\hline \multirow{3}{*}{$\begin{array}{l}\text { Sistemas de } \\
\text { manejo }^{(1)}\end{array}$} & \multicolumn{2}{|c|}{$\mathrm{N}$ total aplicado $\left(\mathrm{kg} \mathrm{ha}^{-1}\right)$} & \multicolumn{12}{|c|}{ Estádios de desenvolvimento } \\
\hline & \multirow[t]{2}{*}{ Semeadura } & \multirow[t]{2}{*}{ Cobertura } & \multicolumn{3}{|c|}{ V3 } & \multicolumn{3}{|c|}{ V6 } & \multicolumn{3}{|c|}{$\mathrm{V} 10$} & \multicolumn{3}{|c|}{ Espigamento } \\
\hline & & & $\overline{\mathrm{TRC}}$ & IS & $\begin{array}{c}\mathrm{N} \text { aplicado } \\
\left(\mathrm{kg} \mathrm{ha}^{-1}\right)\end{array}$ & TRC & IS & $\begin{array}{c}\mathrm{N} \text { aplicado } \\
\left(\mathrm{kg} \mathrm{ha}^{-1}\right)\end{array}$ & TRC & IS & $\begin{array}{c}\mathrm{N} \text { aplicado } \\
\left(\mathrm{kg} \mathrm{ha}^{-1}\right)\end{array}$ & TRC & IS & $\begin{array}{c}\mathrm{N} \text { aplicado } \\
\left(\mathrm{kg} \mathrm{ha}^{-1}\right)\end{array}$ \\
\hline S1 & 0 & 0 & 18,6 & 0,56 & 0 & 28,3 & 0,71 & 0 & 41,6 & 0,77 & 0 & 34,0 & 0,55 & 0 \\
\hline S2 & 10 & 120 & 25,2 & - & 30 & 35,8 & - & 30 & 49,9 & - & 30 & 56,2 & - & 30 \\
\hline S3 & 10 & 120 & - & 0,81 & 30 & - & 0,90 & 30 & - & 0,91 & 30 & - & 0,87 & 30 \\
\hline S4 & 10 & 120 & - & - & 30 & - & - & 30 & - & - & 30 & - & - & 30 \\
\hline S11 & 10 & 120 & 25,7 & - & 30 & 8,7 & $\mathrm{O}_{3}^{-}$ & 30 & 47,6 & - & 30 & 51,5 & - & 30 \\
\hline S5 & 20 & 180 & 27,4 & - & 60 & 37,1 & - & 60 & 51,7 & - & 60 & 59,5 & - & - \\
\hline S6 & 20 & 180 & - & 0,77 & 60 & - & 0,90 & 60 & - & 0,97 & - & - & 0,85 & 60 \\
\hline S7 & 20 & 240 & - & - & 60 & - & - & 60 & - & - & 60 & - & - & 60 \\
\hline $\mathrm{S} 12$ & 20 & 180 & 28,7 & - & 60 & 13,7 & $\mathrm{IO}_{3}^{-}$ & 60 & 53,0 & - & 60 & 58,5 & - & - \\
\hline S8 & 35 & 315 & 29,1 & - & 105 & 40,9 & - & 105 & 54,6 & - & 105 & 61,0 & - & - \\
\hline S9 & 35 & 315 & - & 0,86 & 105 & - & 0,96 & - & - & 0,92 & 105 & - & 0,94 & 105 \\
\hline S10 & 35 & 420 & - & - & 105 & - & - & 105 & - & - & 105 & - & - & 105 \\
\hline $\mathrm{S} 13$ & 35 & 315 & 28,1 & - & 105 & 18,8 & $\mathrm{JO}_{3}^{-}$ & 105 & 55,2 & - & 105 & 60,3 & - & - \\
\hline S14 & 75 & 480 & 33,1 & - & 120 & 39,8 & - & 120 & 54,2 & - & 120 & 61,2 & - & 120 \\
\hline
\end{tabular}

${ }^{(1)}$ S1, testemunha; S2, monitorado-TRCC; S3, monitorado-IS; S4, padrão; S11, monitorado-NO ${ }_{3}^{-}$; S5, monitorado-TRCC; S6, monitorado-IS; S7, padrão; S12, monitorado- $\mathrm{NO}_{3}^{-} ; \mathrm{S} 8$, monitorado-TRCC; S9, monitorado-IS; S10, padrão; S13, monitorado-NO ${ }_{3}^{-}$; $\mathrm{S}_{14}$, referência. 
Nos dois experimentos, não foi aplicada a dose de $\mathrm{N}$ preestabelecida, quando o TRCC e o IS foram superiores, nos sistemas monitorados, ao nível crítico para cada estádio de desenvolvimento e ao IS de 0,95, respectivamente. Esta diferença resultou em variação do rendimento de grãos entre sistemas de manejo.

No Experimento I, o rendimento de grãos foi estatisticamente semelhante entre os sistemas monitorados com base nos TRCC estabelecidos por Argenta (2001) (S2, S5 e S8) e os sistemas padrões, sem monitoramento (S4, S7 e S10), em todas as doses de N (Tabela 3). Com o monitoramento, houve redução, na aplicação, de $60 \mathrm{~kg} \mathrm{ha}^{-1} \mathrm{de} \mathrm{N}$, no S5, e de $105 \mathrm{~kg} \mathrm{ha}^{-1}$ de $\mathrm{N}$, no S8, em relação aos sistemas padrões S7 e S10, respectivamente, sem diminuição no rendimento de grãos. Nos sistemas monitorados com base no IS houve redução de 30 (S3), 120 (S6) e 315 (S9) $\mathrm{kg} \mathrm{ha}^{-1}$ de $\mathrm{N}$ nas doses aplicadas deste nutriente em relação aos sistemas padrões S4, S7 e S10, respectivamente. Contudo, os rendimentos de grãos nos sistemas monitorados com base no IS foram inferiores aos alcançados nos sistemas padrões, sem monitoramento, indicando que a redução da dose neste caso restringiu a produtividade da cultura. Os aumentos no rendimento de grãos e na margem bruta apresentaram comportamento semelhante ao descrito para rendimento de grãos.

Ao se analisar as variáveis eficiências técnica, econômica e de uso do $\mathrm{N}$, verificou-se um novo cenário (Tabela 3). O monitoramento com o IS quase sempre se mostrou igual ou superior ao sistema monitorado com o TRCC e, principalmente, em relação aos sistemas padrões. Assim, apesar de o monitoramento com o IS ter subestimado a necessidade de $\mathrm{N}$ pela planta, que reduziu o rendimento de grãos e os aumentos no rendimento de grãos e na margem bruta, esse método mostrou-se igual ou mais eficiente econômica e tecnicamente, e no uso de $\mathrm{N}$, que os sistemas monitorados com o TRCC e os padrões. Os dois índices de monitoramento (TRCC e IS) apresentaram melhores resultados em termos de eficiências técnica, econômica e de uso do $\mathrm{N}$ que os sistemas padrões, que utilizaram doses de $\mathrm{N}$ preestabelecidas, na maioria das situações estudadas.

No Experimento II, nos sistemas monitorados do grupo de tratamentos com dose média de N (S2, S3 e S11) foram aplicadas as mesmas doses que no sistema padrão (S4), resultando na obtenção de respostas iguais para todas as variáveis avaliadas (Tabela 4). Nos sistemas monitorados do grupo com dose alta de $\mathrm{N}$ (S5, S6 e S12), foram aplicados $60 \mathrm{~kg} \mathrm{ha}^{-1}$ a menos que no sistema padrão (S7), que resultou no rendimento de grãos significativamente menor em relação ao sistema padrão. Por sua vez, nos sistemas monitorados do grupo com dose muito alta de N (S8, S9 e S13) economizou-se uma aplicação de $105 \mathrm{~kg} \mathrm{ha}^{-1}$ de $\mathrm{N}$ em relação ao sistema padrão (S10), sem redução no rendimento de grãos.

Não houve diferença significativa entre os sistemas monitorados e os sistemas padrões para as variáveis

Tabela 3. Rendimento, aumento no rendimento de grãos e margem bruta e eficiência técnica, econômica e do uso do N do híbrido de milho Pioneer 32R21 sob dez sistemas de manejo de N, no experimento $\mathrm{I}^{(1)}$.

\begin{tabular}{|c|c|c|c|c|c|c|c|c|}
\hline $\begin{array}{l}\text { Grupos de tratamentos } \\
\text { com dose de } \mathrm{N}\end{array}$ & $\begin{array}{l}\text { Sistema de } \\
\text { manejo }\end{array}$ & $\begin{array}{l}\mathrm{N} \text { total aplicado } \\
\left(\mathrm{kg} \mathrm{ha}^{-1}\right)\end{array}$ & $\begin{array}{c}\text { Rendimento } \\
\text { de grãos } \\
\left(\mathrm{t} \mathrm{ha}^{-1}\right)\end{array}$ & $\begin{array}{l}\text { Aumento no } \\
\text { rendimento de } \\
\text { grãos }^{(2)}\left(\mathrm{t} \mathrm{ha}^{-1}\right)\end{array}$ & $\begin{array}{c}\text { Aumento na } \\
\text { margem bruta } \\
\left(\mathrm{R} \$ \mathrm{ha}^{-1}\right)\end{array}$ & $\begin{array}{c}\text { Eficiência } \\
\text { técnica }{ }^{(4)} \\
\left(\mathrm{kg} \mathrm{grão} \mathrm{kg} \mathrm{N}^{-1}\right) \\
\end{array}$ & $\begin{array}{c}\text { Eficiência } \\
\text { econômica }^{(5)} \\
\left(\mathrm{R} \$ \text { grão } \mathrm{R} \$ \mathrm{~N}^{-1}\right)\end{array}$ & $\begin{array}{c}\text { Eficiência } \\
\text { do uso do } \mathrm{N}^{(6)} \\
\left(\mathrm{kg}_{\left.\text {grão } \mathrm{kg} \mathrm{N}^{-1}\right)}\right.\end{array}$ \\
\hline \multirow[t]{4}{*}{ Média } & S1 - testemunha & 0 & $6,28 \mathrm{c}$ & - & - & - & - & - \\
\hline & $\mathrm{S} 2$ - monitorado-TRCC & 130 & $12,88 \mathrm{a}$ & $6,60 \mathrm{a}$ & $1.571 \mathrm{a}$ & $50,78 \mathrm{a}$ & 6,25 & $67,19 \mathrm{a}$ \\
\hline & S3 - monitorado-IS & 100 & $10,21 \mathrm{~b}$ & $3,93 b$ & $885 b$ & $39,24 \mathrm{a}$ & 4,87 & $86,75 \mathrm{a}$ \\
\hline & S4 - padrão & 130 & $12,17 \mathrm{a}$ & $5,89 \mathrm{a}$ & $1.371 \mathrm{ab}$ & $45,34 \mathrm{a}$ & 5,58 & $69,61 \mathrm{a}$ \\
\hline \multirow[t]{4}{*}{ Alta } & $\mathrm{S} 1$ - testemunha & 0 & $6,28 \mathrm{c}$ & - & - & - & - & - \\
\hline & S5 - monitorado-TRCC & 200 & $12,27 \mathrm{ab}$ & $6,00 \mathrm{ab}$ & $1.296 \mathrm{a}$ & $29,98 \mathrm{a}$ & 4,21 & $62,64 a b$ \\
\hline & S6 - monitorado-IS & 140 & $10,44 b$ & $4,17 b$ & $901 \mathrm{a}$ & $29,78 \mathrm{a}$ & 4,21 & $71,72 \mathrm{a}$ \\
\hline & S7 - padrão & 260 & $13,31 \mathrm{a}$ & $7,00 \mathrm{a}$ & $1.466 \mathrm{a}$ & $27,04 \mathrm{a}$ & 3,78 & $47,99 \mathrm{~b}$ \\
\hline \multirow[t]{4}{*}{ Muito alta } & $\mathrm{S} 1$ - testemunha & 0 & $6,28 \mathrm{c}$ & - & - & - & - & - \\
\hline & S8 - monitorado-TRCC & 350 & $13,66 \mathrm{a}$ & $7,38 \mathrm{a}$ & $1.427 \mathrm{a}$ & $21,10 \mathrm{~b}$ & 3,15 & $47,21 \mathrm{~b}$ \\
\hline & S9-monitorado-IS & 140 & $10,79 b$ & $4,51 \mathrm{~b}$ & $1.016 \mathrm{a}$ & $32,24 \mathrm{a}$ & 4,87 & $86,84 \mathrm{a}$ \\
\hline & $\mathrm{S} 10$ - padrão & 455 & $13,51 \mathrm{a}$ & $7,24 \mathrm{a}$ & $1.184 \mathrm{a}$ & $15,91 \mathrm{c}$ & 2,37 & $34,38 \mathrm{c}$ \\
\hline
\end{tabular}

${ }^{(1)}$ Médias seguidas pela mesma letra na coluna, dentro do mesmo grupo, não diferem estatisticamente pelo teste de Duncan, a $5 \%$ de probabilidade. ${ }^{(2)}$ Aumento no rendimento de grãos dos tratamentos que receberam $\mathrm{N}$ em relação à testemunha (sem $\mathrm{N}$ ). ${ }^{(3)}$ Aumento na margem bruta: diferença entre ganho monetário com aumento no rendimento de grãos com aplicação de $\mathrm{N}$ (R \$ 17,00/60 kg de milho) e custo do fertilizante (R \$ 785,00/t de uréia) e de sua aplicação (R $\$ 18,13$ por aplicação). ${ }^{(4)}$ (Rendimento de grãos do tratamento com $\mathrm{N}$ - rendimento de grãos da testemunha sem $\left.\mathrm{N}\right) /$ quantidade de $\mathrm{N}$ aplicada. ${ }^{(5)}$ Receita bruta advinda do aumento da produtividade com aplicação de N/custo do fertilizante e de sua aplicação. ${ }^{(6)}$ (Rendimento de grãos da parcela fertilizada com $\mathrm{N}$ - rendimento de grãos da testemunha sem $\left.\mathrm{N}\right) /(\mathrm{N}$ acumulado na planta do tratamento com $\mathrm{N}$ - N acumulado na planta da testemunha sem $\mathrm{N}$ ). 
aumentos no rendimento de grãos e na margem bruta e eficiência técnica, exceto no sistema S6, que apresentou maior eficiência técnica que a dos demais sistemas no grupo de tratamentos com dose alta de N, o que não se justifica, pois foi aplicada a mesma dose de $\mathrm{N}$ em todos os sistemas.

A aplicação de $60 \mathrm{~kg} \mathrm{ha}^{-1}$ de $\mathrm{N}$ a menos nos sistemas monitorados do grupo com dose alta de N (S5, S6 e S12), apesar de ter causado redução no rendimento de grãos em relação ao sistema padrão (S7), não resultou em diferenças significativas entre os sistemas monitorados e o padrão para as variáveis eficiência econômica e de uso do N (Tabela 4). Isto pode ser explicado porque, no cálculo dessas variáveis, são contabilizados o rendimento de grãos e a dose aplicada de N. Desta forma, o menor rendimento de grãos obtido nos sistemas monitorados do grupo com dose alta de $\mathrm{N}$ foi compensado economicamente pela menor dose aplicada em relação ao sistema padrão (S7). Os sistemas monitorados do grupo com dose muito alta de N (S8, S9 e S13), quando se economizou a aplicação de $105 \mathrm{~kg} \mathrm{ha}^{-1} \mathrm{de}$ $\mathrm{N}$ em relação ao sistema padrão (S10), sem redução no rendimento de grãos, apresentaram maiores eficiências econômica e de uso do $\mathrm{N}$ em relação ao sistema padrão (S10).
Os dados evidenciam que níveis críticos do teor relativo de clorofila na folha (TRCC) estabelecidos por Argenta (2001) são eficientes para separar plantas com deficiência e com teor adequado de N. Esta afirmação está fundamentada nos dados de rendimentos de grãos nos sistemas monitorados com os níveis críticos (TRCC), nos dois experimentos (Tabelas 3 e 4). Esses sistemas, mesmo quando receberam doses de $\mathrm{N}$ inferiores à dos sistemas padrões, não diferiram estatisticamente dos sistemas padrões quanto ao rendimento de grãos, exceto no sistema S5, no Experimento II. Além disso, os sistemas monitorados com TRCC sempre apresentaram desempenho igual ou superior aos sistemas padrões quanto as variáveis: aumentos no rendimento de grãos e na margem bruta, e eficiências técnica, econômica e de uso do N (Tabelas 3 e 4). Resultados semelhantes foram registrados por Peng et al. (1996), Balasubramanian et al. (1999) e Singh et al. (2002) com a cultura do arroz. Nestes estudos, os autores observaram maior eficiência da adubação nitrogenada nos sistemas monitorados com clorofilômetro usando níveis críticos do que nos sistemas em que foram aplicadas doses predeterminadas de N. Segundo Peng et al. (1996), isto se deve, principalmente, à maior

Tabela 4. Rendimento, aumento no rendimento de grãos e margem bruta e eficiência técnica, econômica e do uso do N do híbrido de milho Pioneer 32R21 sob 14 sistemas de manejo de N, no experimento II ${ }^{(1)}$.

\begin{tabular}{|c|c|c|c|c|c|c|c|c|}
\hline $\begin{array}{l}\text { Grupos de } \\
\text { tratamentos }\end{array}$ & $\begin{array}{l}\text { Sistema de } \\
\text { manejo }\end{array}$ & $\begin{array}{l}\mathrm{N} \text { total } \\
\text { aplicado } \\
\left(\mathrm{kg} \mathrm{ha}^{-1}\right)\end{array}$ & $\begin{array}{l}\text { Rendimento } \\
\text { de grãos } \\
\left(\mathrm{t} \mathrm{ha}^{-1}\right)\end{array}$ & $\begin{array}{l}\text { Aumento no } \\
\text { rendimento } \\
\text { de grãos } \\
\left(\mathrm{tha}^{-1}\right)\end{array}$ & $\begin{array}{l}\text { Aumento } \\
\text { na margem } \\
\text { bruta }^{(3)} \\
\left(\mathrm{R} \$ \mathrm{ha}^{-1}\right)\end{array}$ & $\begin{array}{c}\text { Eficiência } \\
\text { técnica }^{(4)} \\
\left(\mathrm{kg} \text { grão } \mathrm{kg} \mathrm{N}^{-1}\right)\end{array}$ & $\begin{array}{c}\text { Eficiência } \\
\text { econômica } \\
(5) \\
\left(\mathrm{R} \$ \text { grão } \mathrm{R} \$ \mathrm{~N}^{-1}\right)\end{array}$ & $\begin{array}{c}\text { Eficiência de } \\
\text { uso do } \mathrm{N}^{(6)} \\
\left(\mathrm{kg} \text { grão } \mathrm{kg} \mathrm{N}^{-1}\right)\end{array}$ \\
\hline \multirow[t]{6}{*}{ Alta } & S1 - testemunha & 0 & $5,81 \mathrm{c}$ & - & - & - & -5 & - \\
\hline & $\mathrm{S} 2$ - monitorado-TRCC & 130 & $12,09 \mathrm{~b}$ & $4,35 b$ & $1.131,5 \mathrm{a}$ & $48,31 \mathrm{a}$ & 4,54 & $49,24 a$ \\
\hline & S3 - monitorado-IS & 130 & $11,46 b$ & $5,10 \mathrm{~b}$ & $1.380,0 \mathrm{a}$ & $43,48 \mathrm{a}$ & 5,32 & $50,49 \mathrm{a}$ \\
\hline & S4 - padrão & 130 & $12,06 \mathrm{~b}$ & $5,11 \mathrm{~b}$ & $1.383,4 \mathrm{a}$ & $48,90 \mathrm{a}$ & 5,33 & $57,65 \mathrm{a}$ \\
\hline & $\mathrm{S} 11-$ monitorado- $\mathrm{NO}_{3}^{-}$ & 130 & $11,12 b$ & $4,22 \mathrm{~b}$ & $1.086,3 \mathrm{a}$ & $40,87 \mathrm{a}$ & 4,40 & $62,99 \mathrm{a}$ \\
\hline & S14 - referência & 555 & $15,22 \mathrm{a}$ & $6,99 \mathrm{a}$ & $1.203,5 \mathrm{a}$ & $16,95 b$ & 2,07 & $31,76 \mathrm{~b}$ \\
\hline \multirow[t]{6}{*}{ Alta } & $\mathrm{S} 1$ - testemunha & 0 & $5,81 \mathrm{c}$ & - & - & - & - & - \\
\hline & S5 - monitorado-TRCC & 200 & $12,89 \mathrm{~b}$ & $6,90 \mathrm{a}$ & $1.866,2 \mathrm{a}$ & $35,42 b$ & 5,30 & $47,07 \mathrm{ab}$ \\
\hline & S6 - monitorado-IS & 200 & $13,51 b$ & $6,81 \mathrm{a}$ & $1.837,0 \mathrm{a}$ & $38,51 \mathrm{a}$ & 5,23 & $49,30 \mathrm{ab}$ \\
\hline & S7 - padrão & 260 & $14,74 \mathrm{a}$ & $7,81 \mathrm{a}$ & $2.036,1 \mathrm{a}$ & $34,37 b$ & 4,59 & $41,07 \mathrm{~b}$ \\
\hline & $\mathrm{S} 12-$ monitorado- $\mathrm{NO}_{3}{ }^{-}$ & 200 & $12,81 \mathrm{~b}$ & $6,39 \mathrm{a}$ & $1.697,0 \mathrm{a}$ & $35,02 b$ & 4,91 & $51,73 a$ \\
\hline & S14-referência & 555 & $15,22 \mathrm{a}$ & $6,99 \mathrm{a}$ & $1.203,5 \mathrm{~b}$ & $16,95 \mathrm{c}$ & 2,07 & $31,76 \mathrm{c}$ \\
\hline \multirow[t]{6}{*}{ Muito alta } & $\mathrm{S} 1$ - testemunha & 0 & $5,81 \mathrm{c}$ & - & - & - & 219 & - \\
\hline & S8 - monitorado- TRCC & 350 & $14,84 \mathrm{a}$ & $7,38 \mathrm{a}$ & $1.742,5 \mathrm{a}$ & $25,81 \mathrm{a}$ & 3,42 & $40,16 \mathrm{a}$ \\
\hline & S9 - monitorado-IS & 350 & $14,82 \mathrm{a}$ & $7,19 a$ & $1.676,2 \mathrm{a}$ & $25,76 \mathrm{a}$ & 3,33 & $37,31 \mathrm{a}$ \\
\hline & $\mathrm{S} 10$ - padrão & 455 & $15,14 \mathrm{a}$ & $7,19 \mathrm{a}$ & $1.458,4 \mathrm{a}$ & $20,50 \mathrm{~b}$ & 2,55 & $32,54 \mathrm{~b}$ \\
\hline & $\mathrm{S} 13-$ monitorado- $\mathrm{NO}_{3}{ }^{-}$ & 350 & $14,53 \mathrm{a}$ & $7,10 \mathrm{a}$ & $1.645,7 \mathrm{a}$ & $24,92 \mathrm{a}$ & 3,29 & $39,42 \mathrm{a}$ \\
\hline & S14-referência & 555 & $15,22 \mathrm{a}$ & $6,99 a$ & $1.203,5 \mathrm{a}$ & $16,95 \mathrm{c}$ & 2,07 & $31,76 \mathrm{~b}$ \\
\hline
\end{tabular}

${ }^{(1)}$ Médias seguidas pela mesma letra na coluna, dentro do mesmo grupo, não diferem estatisticamente pelo teste de Duncan, a $5 \%$ de probabilidade. ${ }^{(2)}$ Aumento no rendimento de grãos dos tratamentos que receberam $\mathrm{N}$ em relação à testemunha (sem $\left.\mathrm{N}\right)$. ${ }^{(3)} \mathrm{Aumento}$ na margem bruta: diferença entre ganho monetário com aumento no rendimento de grãos com aplicação de N (R\$17,00/60 kg de milho) e custo do fertilizante (R\$ 785/t de uréia) e de sua aplicação [R $\$ 18,13 /$ aplicação, de acordo com Federação das Cooperativas Agropecuárias do Rio Grande do Sul (2007)]. (4)(Rendimento de grãos do tratamento com N - rendimento de grãos da testemunha sem N)/quantidade de $\mathrm{N}$ aplicada. ${ }^{(5)}$ Receita bruta advinda do aumento da produtividade com aplicação de $\mathrm{N} /$ custo do fertilizante e de sua aplicação. ${ }^{(6)}$ (Rendimento de grãos da parcela fertilizada com $\mathrm{N}$ - rendimento de grãos da testemunha sem $\mathrm{N}) /(\mathrm{N}$ acumulado na planta do tratamento com $\mathrm{N}-\mathrm{N}$ acumulado na planta da testemunha sem $\mathrm{N})$. 
sincronia entre a época de aplicação de $\mathrm{N}$ e a sua demanda da planta.

No que se refere à utilização do IS de 0,95 como critério para monitoramento do teor de $\mathrm{N}$ na planta, observou-se que a resposta variou nos dois experimentos. No Experimento I, esse índice não foi eficiente para separar plantas com deficiência das com teor adequado de $\mathrm{N}$. Isto pode ser verificado pelos menores rendimentos de grãos obtidos nos sistemas monitorados com IS em relação aos sistemas padrões (Tabela 3). Esta resposta pode ser devida a dois fatores.

Primeiramente, neste experimento não se implementou uma parcela específica de referência, utilizando-se a parcela que apresentava maior valor na leitura do TRC em cada estádio como referência. Assim, pode ter ocorrido superestimação do IS em razão da leitura na parcela de referência ter sido muito próxima da leitura nas parcelas que receberam os demais níveis de $\mathrm{N}$, evidenciando a ausência de necessidade de aplicação de $\mathrm{N}$ em condições em que este nutriente era necessário para a planta. Esta hipótese foi confirmada no Experimento II, em que foi estabelecida uma parcela de referência específica para determinação do IS, e o monitoramento com o IS de 0,95 apresentou eficiência similar ao com base nos níveis críticos.

Além disso, foi utilizado um único IS em todos os estádios de desenvolvimento. É possível que o IS crítico, da mesma forma que ocorre com o TRCC, varie durante a ontogenia da planta de milho. Godoy et al. (2003) sugerem que sejam adotados IS diferentes durante o ciclo do milho, utilizando os valores de 0,98 (V4), 0,95 (V7) e 0,90 (próximo ao pendoamento). Alguns autores recomendam a utilização de outros IS críticos, dependendo do estádio de desenvolvimento da cultura. De acordo com Sainz Rozas \& Echeverría (1998), um IS entre 0,97 e 0,98 deve ser mantido a partir do estádio V6 para se alcançar $95 \%$ do rendimento de grãos máximo. Em contrapartida, Jemison \& Lytle (1996) postulam que valores de IS abaixo de 0,93 indicam situações de deficiência de $\mathrm{N}$, corroborando resultados de Piekielek et al. (1995) que recomendam este mesmo valor para o estádio de grãos leitosos. Contudo, Varvel et al. (1997) sustentam que se o IS for menor que 0,90 no estádio $\mathrm{V} 8$, a deficiência de $\mathrm{N}$ não poderá ser corrigida posteriormente com adubação em cobertura para maximizar o rendimento de grãos.

Apesar de o monitoramento com o IS não ter sido eficiente para atingir os mesmos rendimentos de grãos obtidos nos sistemas padrões, ele proporcionou a obtenção de eficiências técnica, econômica e de uso do $\mathrm{N}$ iguais ou superiores às dos respectivos sistemas padrões (Tabela 3). Isto é muito importante para a agricultura moderna, em que se busca maximizar o uso dos recursos do ambiente.

No Experimento II, as duas formas de monitoramento foram eficientes em separar plantas com deficiência das com teor adequado de $\mathrm{N}$, nos grupos com dose média e muito alta de $\mathrm{N}$. Isto ficou confirmado pela obtenção de rendimentos de grãos, nestes sistemas monitorados, estatisticamente iguais aos dos respectivos padrões (Tabela 4). No grupo de dose alta, o rendimento de grãos dos sistemas monitorados foi inferior ao do sistema padrão. Porém, neste grupo, os aumentos no rendimento de grãos e na margem bruta e as eficiências técnica, econômica e de uso do $\mathrm{N}$ nos sistemas monitorados foram iguais ou superiores ao sistema padrão, o que também foi verificado nos grupos de dose média e muito alta, demostrando a eficiência dos sistemas monitorados (Tabelas 3 e 4).

Resultados similares foram obtidos por Hussain et al. (2000) na cultura do arroz irrigado. Eles observaram maior eficiência agronômica da adubação nitrogenada quando foi utilizado o IS em relação ao uso de doses preestabelecidas no manejo do $\mathrm{N}$, em várias cultivares e em dois locais. Estes mesmos autores concluíram que o IS se adapta a diferentes estações de crescimento, tipos de solo e cultivares. Godoy et al. (2003) também obtiveram resultados favoráveis utilizando o IS como critério para definição de quando aplicar o $\mathrm{N}$ em cobertura no milho. Esses autores concluíram que o IS permitiu definir quando se deveria aplicar o $\mathrm{N}$ em cobertura a partir do estádio de sete a oito folhas.

O monitoramento integrado usando o teor de nitrato no solo (V6) e o TRCC (demais estádios) não se diferenciou do monitoramento somente com o TRCC ou IS. Apesar destes resultados, a estratégia de uso integrado dessas características não deve ser descartada, visto que não houve diferença estatística, nas variáveis avaliadas, entre os sistemas monitorados somente com clorofilômetro e os sistemas monitorados integrando o teor de nitrato no solo e o clorofilômetro (Tabela 4). Além disso, esses resultados não podem ser considerados conclusivos, pois são oriundos de apenas um experimento e um ano. Há necessidade de mais estudos para explorar e concluir sobre a integração entre características de solo e de planta no monitoramento do teor de N no sistema solo- 
planta para predizer a necessidade de sua aplicação em cobertura.

Esperava-se que o monitoramento integrando uma característica de solo (nitrato no solo), especificamente no estádio V6, com uma característica de planta (teor relativo de clorofila na folha), nos demais estádios, apresentasse melhor resultado que o realizado somente com esta característica de planta durante toda a ontogenia. Isto porque, segundo Klapwyk \& Ketterings (2006), a determinação do teor de nitrato no solo neste estádio do milho, que é chamado de teste de pré-aplicação de N em cobertura ("Pre-sidedress Nitrate Test"), é atualmente a melhor ferramenta disponível para os produtores da região Nordeste dos Estados Unidos da América (EUA) para determinar se o milho responderá à aplicação de $\mathrm{N}$ em cobertura. Este teste tem sido muito estudado, principalmente nos EUA, pois permite avaliar a variação da disponibilidade de $\mathrm{N}$ do solo durante o ciclo da cultura, determinando maior flexibilidade no manejo da adubação nitrogenada em situações específicas (Rambo et al., 2004). Além disso, pressupõe-se que com a integração entre diferentes parâmetros para auxiliar o manejo da adubação nitrogenada em milho, eles sejam utilizados de forma complementar, antes e durante a sua ontogenia, de forma que um atributo complemente as características ou supra as deficiências de outro (Rambo et al., 2004).

Considerando os resultados obtidos nos dois anos de estudo, pode-se afirmar que os monitoramentos com o TRCC e com o IS são alternativas viáveis para refinamento do manejo do $\mathrm{N}$ em cobertura em milho. Contudo, o uso dos dois índices têm suas vantagens e desvantagens.

No que diz respeito ao uso do TRCC, salienta-se que este trabalho foi realizado no mesmo local e com o mesmo híbrido de milho utilizado por Argenta (2001) e sob condições de alta fertilização, controle de plantas daninhas, pragas e doenças adequado e com irrigação. Assim, deve-se questionar se os TRCC propostos por este autor apresentarão a mesma eficiência quando utilizados em outros locais, com outros híbridos e em condições de manejo menos apropriadas, pois outros fatores, além da disponibilidade de $\mathrm{N}$ no solo, podem afetar a intensidade da coloração verde da folha e a respectiva leitura pelo clorofilômetro (Waskom et al., 1996; Sunderman et al., 1997; Bredemeier, 1999).

A utilização do IS tem sido recomendada justamente para diminuir a influência dos fatores acima citados na leitura do clorofilômetro (Waskom et al., 1996; Hussain et al., 2000; Costa et al., 2001). O uso do IS no manejo da adubação nitrogenada tem sido associado ao monitoramento do teor de $\mathrm{N}$ na planta, na estratégia denominada de adubar quando necessário, efetivada especialmente sob condições de fertirrigação (Blackmer \& Schepers, 1994; Varvel et al., 1997). O uso deste índice também tem sido testado na predição de doses economicamente ótimas de $\mathrm{N}$ em milho (Scharf et al., 2006).

Contudo, uma questão importante referente ao uso do IS é que, mesmo com o uso de faixas de referência, há necessidade de se definir qual deve ser o valor mais adequado a cada estádio de desenvolvimento da planta de milho. Além disso, ainda não existem critérios estabelecidos para definição da dose e época de aplicação de $\mathrm{N}$ a ser aplicada na faixa de referência. Assim, se estas faixas não forem manejadas corretamente, a necessidade de aplicação de $\mathrm{N}$ em cobertura pode ser subestimada ou superestimada, reduzindo o rendimento de grãos ou aumentando a contaminação ambiental, respectivamente (Sunderman et al., 1997). Outro problema potencial é que muitos agricultores podem relutar em manejar uma faixa da lavoura de forma diferenciada, aplicando dose mais elevada de $\mathrm{N}$ na faixa de referência.

Em síntese, a escolha do índice (TRCC ou IS) a ser utilizado no monitoramento do nível de $\mathrm{N}$ na planta com o clorofilômetro irá depender dos fatores discutidos anteriormente. Pressupõe-se que, quando há disponibilidade de índices TRCC para o híbrido utilizado, adequados para a região em que se está trabalhando, seria mais conveniente a opção por esses índices. Na ausência destes índices críticos regionais para o híbrido utilizado, a melhor opção seria o uso do IS.

\section{Conclusões}

1. A eficiência para identificar plantas de milho deficientes e com teor adequado de $\mathrm{N}$ pelos métodos de monitoramento do seu teor na planta, com base no teor relativo de clorofila na folha crítico (desenvolvido para a mesma cultivar, local e condições de manejo em estudo) e no índice de suficiência $(0,95)$ é similar.

2. O monitoramento do teor de $\mathrm{N}$ na planta em milho com base no teor relativo de clorofila na folha, medido pelo clorofilômetro, aumenta a eficiência de uso do $\mathrm{N}$, em relação ao sistema de manejo não monitorado, quando são utilizadas altas doses deste nutriente. 
3. O monitoramento com base na integração entre o teor de nitrato no solo e o teor relativo crítico de clorofila na folha não é mais eficiente do que o monitoramento com base somente no teor relativo crítico de clorofila na folha ou no índice de suficiência, no ambiente avaliado.

\section{Agradecimentos}

Ao CNPq, pelas bolsas de pesquisa e pelo financiamento parcial deste trabalho.

\section{Referências}

AMADO, T.J.C.; MIELNICZUK, J.; AITA, C. Recomendação de adubação nitrogenada para o milho no RS e SC adaptada ao uso de culturas de cobertura do solo, sob sistema de plantio direto. Revista Brasileira de Ciência do Solo, v.26, p.241-248, 2002.

ARGENTA, G. Monitoramento do nível de nitrogênio na planta como indicador da adubação nitrogenada em milho. 2001. 112p. Tese (Doutorado) - Universidade Federal do Rio Grande do Sul, Porto Alegre.

ARGENTA, G.; SILVA, P.R.F. da; SANGOI, L. Leaf relative chlorophyll content as an indicator parameter to predict nitrogen fertilization in maize. Ciência Rural, v.34, p.1379-1387, 2004.

BALASUBRAMANIAN, V.; MORALES, A.C.; CRUZ, R.T.; ABDULRACHMAN, S. On-farm adaptation of knowledge-intensive nitrogen management technologies for rice systems. Nutrient Cycling in Agroecosystems, v.53, p.59-69, 1999.

BLACKMER, T.M.; SCHEPERS, J.S. Techniques for monitoring crop nitrogen status in corn. Communications in Soil Science and Plant Analysis, v.25, p.1791-1800, 1994.

BLACKMER, T.M.; SCHEPERS, J.S. Use of a chlorophyll meter to monitor nitrogen status and schedule fertigation for corn. Journal of Production Agriculture, v.8, p.56-60, 1995.

BREDEMEIER, C. Predição da necessidade de nitrogênio em cobertura em trigo e aveia. 1999. 101p. Dissertação (Mestrado) Universidade Federal do Rio Grande do Sul, Porto Alegre.

COSTA, C.; DWYER, L.M.; DUTILLEUL, P.; STEWART, D.W.; MA, B.L.; SMITH, D.L. Inter-relationships of applied nitrogen, SPAD, and yield of leafy and non-leafy maize genotypes. Journal of Plant Nutrition, v.24, p.1173-1194, 2001.

EMBRAPA. Centro Nacional de Pesquisa de Solos (Rio de Janeiro, RJ). Sistema brasileiro de classificação de solos. Rio de Janeiro: Embrapa-CNPS, 1999. 412p.

FEDERAÇÃO DAS COOPERATIVAS AGROPECUÁRIAS DO RIO GRANDE DO SUL (Porto Alegre, RS). Evolução dos preços mínimos de soja, milho e trigo. Disponível em: <http:// www.redeagro.com.br/sig/home/modulo.textos.php?link=10>. Acesso em: 15 jan. 2007.
GODOY, L.J.G. de; VILLAS BÔAS, R.L.; GRASSI FILHO, H. Adubação nitrogenada na cultura do milho baseada na medida do clorofilômetro e no índice de suficiência em nitrogênio (ISN). Acta Scientiarum: Agronomy, v.25, p.373-380, 2003.

HUSSAIN, F.; BRONSON, K.F.; SINGH, Y.; SINGH, B.; PENG, S. Use of chlorophyll meter sufficiency indices for nitrogen management of irrigated rice in Asia. Agronomy Journal, v.92, p.875-879, 2000.

INDICAÇÕES técnicas para a cultura de milho e sorgo no Estado do Rio Grande do Sul. Porto Alegre: Fepagro, 2005. 155p.

JEMISON, J.M.; LYTLE, D.E. Field evaluation of two nitrogen testing methods in maize. Journal of Production Agriculture, v.9, p.106-113, 1996.

KLAPWYK, J.H.; KETTERINGS, Q.M. Soil tests for predicting corn response to nitrogen fertilizer in New York. Agronomy Journal, v.98, p.675-681, 2006.

MA, B.L.; DWYER, L.M. Within plot variability in available soil mineral nitrogen in relation to leaf greenness and yield. Communications in Soil Science and Plant Analysis, v.30, p.1919-1928, 1999.

MA, B.L.; MORRISON, M.J.; VOLDENG, H.D. Leaf greenness and photosynthetic rates in soybean. Crop Science, v.35, p.14111414, 1995.

PENG, S.; GARCIA, F.V.; LAZA, R.C.; SANICO, A.L.; VISPERAS, R.M.; CASSMAN, K.G. Increased N-use efficiency using a chlorophyll meter on high-yielding irrigated rice. Field Crop Research, v.47, p.243-252, 1996.

PIEKIELEK, W.P.; FOX, R.H.; TOTH, J.D.; MacNEAL, K.E. Use of a chlorophyll meter at the early dent stage of corn to evaluate nitrogen sufficiency. Agronomy Journal, v.87, p.403-408, 1995.

RAMBO, L.; SILVA, P.R.F. da; ARGENTA, G.; BAYER, C. Testes de nitrato no solo como indicadores complementares no manejo da adubação nitrogenada em milho. Ciência Rural, v.34, p.1279-1287, 2004.

RAUN, W.R.; JOHNSON, G.V. Improving nitrogen use efficiency for cereal production. Agronomy Journal, v.91, p.357-363, 1999.

ROTH, G.W.; BEEGLE, D.B.; FOX, R.H.; TOTH, J.D.; PIEKIELEK, W.P. Development of a quicktest kit method to measure soil nitrate. Communications in Soil Science and Plant Analysis, v.22, p.191-200, 1991.

SAINZ ROZAS, H.; ECHEVERRÍA, H.E. Relación entre las lecturas del medidor de clorofila (Minolta SPAD 502) en distintos estadios del ciclo del cultivo de maíz y el rendimiento en grano. Revista de la Facultad de Agronomía, v.103, p.37-44, 1998.

SCHARF, P.C.; BROUDER, S.M.; HOEFT, R.G. Chlorophyll meter readings can predict nitrogen need and yield response of corn in the North-Central USA. Agronomy Journal, v.98, p.655-665, 2006. 
SIMS, J.T.; VASILAS, B.L.; GARTLEY, K.L.; MILLIKEN, B.; GREEN, V. Evaluation of soil and plant nitrogen tests for maize on manured soils of the Atlantic Coastal-Plain. Agronomy Journal, v.87, p.213-222, 1995.

SINGH, B.; SYNGH, Y.; LADHA, J.K.; BRONSON, K.F.; BALASUBRAMANIAN, V.; SINGH, J.; KHIND, C.S. Chlorophyll meter and leaf color chart based nitrogen management for rice and wheat in northwestern India. Agronomy Journal, v.94, p.821$829,2002$.

SUNDERMAN, H.D.; PONTUS, J.S.; LAWLESS, J.R. Variability in leaf chlorophyll concentration among fully-fertilized corn hy- brids. Communications in Soil Science and Plant Analysis, v.28, p.1793-1803, 1997.

TEDESCO, M.J.; GIANELLO, C.; BISSANI, C.A. Análise de solo, plantas e outros materiais. 2.ed. Porto Alegre: Departamento de Solos da UFRGS, 1995. 174p. (Boletim técnico de solos, 5).

VARVEL, G.E.; SCHEPERS, J.S.; FRANCIS, D.D. Ability for inseason correction of nitrogen deficiency in corn using chlorophyll meters. Soil Science Society of America Journal, v.61, p.12331239, 1997.

WASKOM, R.M.; WESTFALL, D.G.; SPELLMAN, D.E.; SOLTANPOUR, P.N. Monitoring nitrogen status of corn with a portable chlorophyll meter. Communications in Soil Science and Plant Analysis, v.27, p.545-560, 1996. 